\title{
Zwischen Theorie und Deutung der Vererbung psychischer Störungen
}

\section{Zur Übertragung des Mendelismus auf die Psychiatrie in Deutschland und in den USA, 1911-1930}

\section{Anne Cottebrune}

Between Theory and Interpretation of the Hereditary Transmission Process of Mental Disease. The Introduction of Mendelism in German and North American Psychiatry, 1911-1930

\begin{abstract}
1911 saw the beginning of decisive developments in psychiatric genetic research. During that year, two expert papers dealing with the application of the Mendelian Theory were published in Germany and in the United States. Only a decade after the "rediscovery" of the Mendelian Laws simultaneous efforts were being made to better understand the hereditary transmission process of mental diseases by means of the Mendelian Theory. The results of these efforts were disparate. While in the United States, the Mendelian theory was used to support the polymorphous theory of the hereditary transmission process of mental diseases, by which a common hereditary origin of mental diseases was assumed, the introduction of Mendelism in psychiatry in Germany focused on corroborating Emil Kraepelin's concept of a disease entity. The Mendelian Theory especially helps to underpin the idea of a specific genetic origin of the clinical diseases described by Kraepelin. As German and North American psychiatric genetics were inspired by diverse disease and genetic concepts, so too was their use of the Mendelian Theory very divergent. Research linked to the use of the Mendelian Theory was both dissimilar and hotly contested. This was due to the fact that it was difficult, if not impossible to identify Mendelian patterns by studying the hereditary transmission process. As a result, the approach of studying the genetic basis of mental diseases was further developed in Germany.
\end{abstract}

Keywords: psychiatric genetics, mendelism, polymorphismous theory, Ernst Rüdin, Aaron J. Rosanoff

Schlüsse/wörter: Psychiatrische Genetik, Mendelismus, polymorphe Vererbung, Ernst Rüdin, Aaron J. Rosanoff

Die beschleunigte Industrialisierung während des letzten Drittels des 19. Jahrhunderts, die damit zusammenhängenden gesellschaftlichen Veränderungen und die gleichzeitige Entstehung einer modernen Vererbungswissenschaft waren in den USA und in Deutschland für das Verständnis von psychischen Erkrankungen und die Herangehensweisen an sie von enormer Bedeutung. Der industrielle Aufschwung, der gewaltige Zustrom von Immigranten und das damit verbundene Wachstum der Städte brachten in den USA eine Reihe von ethnischen, religiösen und ökonomischen Problemen 
mit sich. In diesem Kontext wurden psychisch Kranke und vor allem sogenannte Schwachsinnige zunehmend als eine Gefahr für die Gesellschaft angesehen. Die Auffassung, die Vererbung spiele bei der Entstehung von psychischen Störungen die Hauptrolle, fand vor dem Hintergrund der Verbreitung biologistischen Gedankenguts immer mehr Resonanz. Mit der eugenischen Bewegung, die sich in den USA rasch etablierte, erhielten Vererbungstheorien einen immer größeren Stellenwert bei der Beschäftigung mit der Ätiologie psychischer Störungen (Rushton 1994: 20-27). Auch in Deutschland waren der Industrialisierungsprozess sowie die städtische Verdichtung und ihre gesellschaftlichen Folgen in dieser Zeit mit einem Umdenken bezüglich des Problems geistiger Erkrankung verbunden. Die Frage nach den Ursachen spielte bei der Beschäftigung mit psychischen Krankheiten eine immer größere Rolle. Seit Mitte des 19. Jahrhunderts wurde die Psychiatrie als eigenständige Disziplin in der Medizin aufgewertet und fand verstärkt Anschluss an den Universitätsbereich. Mit ihrer Institutionalisierung im akademischen Bereich ging ein naturwissenschaftliches Selbstverständnis einher, das sich seit den achtziger Jahren des 19. Jahrhunderts in verstärkten Bemühungen um eine morphologisch-neuropathologische Erforschung der Geistes- und Nervenkrankheiten ausdrückte. Infolgedessen wurde die im Laufe des 19. Jahrhunderts noch verbreitete Annahme, dass psychische Erkrankungen durch Affekte und stark affektiv erregende Erlebnisse verursacht würden, zunehmend verdrängt. Die Enttäuschung über die geringen Erkenntnisse durch neuropathologische Ansätze führte seit etwa der Wende zum 20. Jahrhundert zu einer zunehmenden Fokussierung auf die Rolle der Vererbung bei der Entstehung psychischer Störungen.

Im ausgehenden 19. Jahrhundert waren sowohl deutsche als auch nordamerikanische Psychiater bereits intensiv mit Erblichkeitsfragen beschäftigt. Als mit dem beginnenden 20. Jahrhundert ein breiter Rezeptionsprozess der Mendel'schen Theorie ansetzte, waren sie um eine entsprechende Deutung der Vererbung von psychischen Störungen bemüht. Auch wenn am Anfang ihre Motive, den Mendelismus in die Psychiatrie einzuführen, ähnlich waren, war ihre jeweilige Anwendung sehr unterschiedlich.

Eine genaue Analyse ihrer Forschungsarbeiten zeigt, dass dies am Einfluss divergenter Vererbungs- und Krankheitskonzepte lag. Während in Deutschland die Anwendung des Mendelismus unter dem Einfluss von Kraepelins Krankheitslehre weitgehend mit der Vorstellung klar abgrenzbarer Krankheiten mit je einem spezifischen Erbgang verknüpft wurde, war man in den USA von dieser Vorstellung weit entfernt und suchte - wie im Folgenden zu zeigen sein wird - nach einer gemeinsamen Erbanlage für Geistes- und Nervenkrankheiten. Die ersten Forscher, die in den USA die Vererbung von psychischen Störungen mendelistisch zu deuten versuchten und die hauptsächlich aus der Arbeitsgruppe um den Psychiater Aaron J. Rosanoff stammten, hielten an der Theorie der polymorphen Vererbung psy- 
chischer Krankheiten fest, die seit Mitte des 19. Jahrhunderts eine besondere Förderung durch die rasche Ausbreitung der Degenerationslehre des französischen Psychiaters Benedict Augustin Morel erhalten hatte (Gausemeier 2005: 198-199, Ackerknecht 1957: 51-55). Diese Lehre basierte auf der Idee, dass somatische und psychologische Anomalien, die Morel als „Degenerationen" bezeichnete, sich verstärkten, indem sie auf nachfolgende Generationen übertragen wurden. Indem Morel die verschiedenartigen Krankheiten in einer Generation auf andersgeartete in der vorhergehenden Generation zurückführte, entwickelte er ein Konzept der erblichen Prädisposition, in dem die Annahme einer gemeinsamen erbbiologischen Grundlage von Nerven- und Geisteskrankheiten nahegelegt wird. In Deutschland wurde es seit Ende des 19. Jahrhunderts zunehmend verdrängt, denn es widersprach der in den 1880er Jahren vom Psychiater Emil Kraepelin entwickelten Krankheitslehre und der damit zusammenhängenden These der spezifischen Verursachung von Krankheiten (Sioli 1885).

Kraepelins Krankheitslehre, die nach der Jahrhundertwende zum dominierenden Paradigma deutschsprachiger Psychiatrie geriet (Roelcke 2002a, 2003, Engstrom 1990), war im Kontext der Bemühungen der akademischen Psychiatrie um eine adäquate Bezeichnung der psychischen Störungen und Gruppierung der klinisch beobachtbaren "Symptomencomplexe“ zu sinnvollen Krankheitskategorien entstanden. Sie war aber auch eine Reaktion auf das von Wilhelm Griesinger entwickelte Krankheitsmodell der „Einheitspsychose“ (Engstrom 1990). Griesinger, der Geisteskrankheiten als Gehirnkrankheiten definiert hatte und in Deutschland eine Pionierrolle in Bezug auf die akademische Institutionalisierung der Psychiatrie einnahm, betrachtete die Vielfalt psychischer Symptome als verschiedene Stadien einer einzigen Krankheit. Kraepelin lehnte diese Auffassung dagegen ab und war bemüht, das Modell der spezifischen Krankheitsursache, das sich in den 1870er Jahren im Bereich der Bakteriologie durchgesetzt hatte, auf die Psychopathologie zu übertragen. Seine Krankheitslehre basierte auf der Vorstellung von klar abgrenzbaren, durch die Biologie des Gehirns dominierten Krankheitseinheiten. Sie waren mit dem Postulat einer spezifischen Krankheitsverursachung, spezifischer pathologischer Veränderungen im Gehirngewebe und spezifischer klinischer Erscheinungsformen verbunden (Roelcke 2003).

Symptomatisch für die unterschiedliche Deutung der Vererbung psychischer Störungen in Deutschland und in den USA war die Kritik des Münchner Psychiaters Ernst Rüdin an seinem nordamerikanischen Kollegen Charles C. Davenport. Seit der Gründung der Deutschen Forschungsanstalt für Psychiatrie (DFA) 1917 in München leitete Rüdin die dort angesiedelte Genealogisch-Demographische Abteilung (GDA). ${ }^{1}$ Der Biologe Davenport war Gründer eines 1910 eröffneten Forschungslabors in Cold Spring Harbor nahe New York, des Eugenics Record Office (ERO), in dem die Umsetzung der erworbenen Erkenntnisse über die Vererbung menschlicher Merkmale 
in eugenische Maßnahmen vorbereitet wurde (Allen 1986). Obwohl Rüdin und Davenport beide Leiter von Forschungseinrichtungen waren, die das Ziel verfolgten, die Untersuchung der menschlichen Vererbung in den Dienst eugenischer Ziele zu stellen und im Kampf um die Anerkennung eugenischer Konzepte in der internationalen Arena sehr verbunden waren ${ }^{2}$, wandten sie die Mendel'sche Theorie sehr unterschiedlich an. Rüdin, der bei seiner Deutung der Vererbung psychischer Störungen auf die Vorstellung von klar abgrenzbaren Krankheitseinheiten mit einer je spezifischen Ätiologie zurückgriff, warf Davenport vor allem dessen Nichtbeachtung der von Kraepelin eingeführten Klassifikation und Krankheitslehre bei der Übertragung Mendel'scher Vererbungsmodelle auf die Psychiatrie vor. Im Gegensatz zu Kraepelin vertrat Davenport die Ansicht, dass zu einer bestimmten biologischen Krankheitseinheit unterschiedliche klinische Krankheitsbilder beziehungsweise Symptomenkomplexe gehörten, die aber nicht als solche vererbt werden konnten (Davenport/Weeks 1911, Davenport/Muncey 1916: 204207). Auch wenn er die Theorie der polymorphen Vererbung von Nervenund Geisteskrankheiten, wonach diese auf eine gemeinsame erbbiologische Grundlage zurückgeführt wurden, nicht explizit vertrat, schloss Davenport mit seiner Vorstellung der gesonderten Vererbung von Symptomen eine erbbiologisch begründete Abhängigkeit zwischen unterschiedlichen Krankheitsformen nicht aus. Die von Rüdin formulierte Kritik an Davenports Deutung der Vererbung psychischer Störungen machte also deutlich, wie der Umgang mit dem Mendelismus jeweils von unterschiedlichen Vorstellungen über die Erblichkeit von Nerven- und Geisteskrankheiten geprägt wurde. Insgesamt soll im Folgenden gezeigt werden, wie die Mendel'sche Theorie in den USA und in Deutschland auf verschiedene Weise und mit unterschiedlichem Ausgang in die Psychiatrie eingeführt wurde. Dabei wird insbesondere der entscheidende Einfluss von bereits bestehenden Vererbungs- und Krankheitskonzepten veranschaulicht.

\section{Die Einführung des Mendelismus in die deutsche und nordamerikanische Psychiatrie}

An der Wende vom 19. zum 20. Jahrhundert setzte ein breiter Rezeptionsprozess der von dem österreichischen Augustiner und Naturforscher Gregor Mendel dreißig Jahre zuvor durchgeführten Kreuzungsexperimente ein. In der Historiographie gilt die sogenannte Wiederentdeckung der Mendel'schen Erbgesetze um 1900, die den drei Botanikern Hugo de Vries, Eric von Tschermak-Seysenegg und Carl Correns zugeschrieben wird, als Geburtsstunde der klassischen Genetik (Rheinberger 2006: 75, Olby 1994). Nachdem die Mendel'schen Erbgesetze ein Jahrzehnt lang vor allem an Pflanzen und Tieren bestätigt und in der züchterischen Praxis angewandt 
worden waren, interessierten sich Psychiater dafür, ihre bereits vorhandenen Vorstellungen über die Erblichkeit von psychischen Störungen durch Anwendung der Mendel'schen Gesetze zu überprüfen und nach Möglichkeit zu konkretisieren.

Bei Betrachtung der Motive, die zur Übertragung von Mendels Theorie auf die Psychiatrie in Deutschland und in den USA führten, fällt ihre Ähnlichkeit auf. 1911 erschienen sowohl in den USA als auch in Deutschland die ersten Fachtexte, die das theoretische Grundgerüst für eine Übertragung auf die Psychiatrie lieferten. Mit seinen Forschungsassistenten am Kings Park State Hospital in New York publizierte Rosanoff zwei Aufsätze, in denen er die Stammbäume seiner Patienten anhand der Mendel'schen Theorie zu deuten versuchte (Cannon/Rosanoff 1911, Rosanoff/Orr 1911). In der deutschen Zeitschrift für die gesamte Neurologie und Psychiatrie erläuterte Rüdin in einem längeren programmatischen Aufsatz (1911), wie eine Anwendung der Mendel'schen Theorie bei der Erforschung der Vererbung psychischer Störungen gestaltet werden könne. Somit entwarf er die Konturen eines psychiatrisch-genetischen Forschungskonzepts, das in den folgenden Jahren an der von ihm geleiteten GDA der DFA umgesetzt wurde.

Die zeitgleichen Bemühungen um eine Übertragung des Mendelismus auf die Psychiatrie in den USA und in Deutschland bauten zunächst auf dem Postulat auf, dass psychische Eigenschaften denselben Vererbungsgesetzen wie physische Merkmale gehorchen mussten. Bereits wenige Jahre nach der "Wiederentdeckung“ der Mendel'schen Erbgesetze war man bemüht gewesen, für einige menschliche Merkmale wie die Haar- und Augenfarbe, aber auch für Anomalien wie die Alkaptonurie oder die Brachydaktilie einen entsprechenden Vererbungsvorgang nachzuweisen. ${ }^{3}$ Damit wurde das Ziel verfolgt, die vorausgesetzte Gültigkeit der Gesetze beim Menschen zu bestätigen. Abbildung 1 stammt aus der 1912 erschienenen Monographie von Davenport mit dem Titel Heredity in Relation to Eugenics und gibt in Form einer Tabelle die bei Augenfarbenanalysen hervorgegangenen Befunde wieder, die zur Annahme einer Mendel'schen Vererbung der Augenfarbe führten. Davon lässt sich die Rezessivität der Augenfarbe blau entnehmen.

Mit seinen Forschungen zur Vererbung geistiger Erkrankungen war auch Rosanoff von vornherein bestrebt, die Wirkung von Mendels Gesetze auf diesem Feld nachzuweisen. In der Einleitung zu seinem Aufsatz von 1911 im Journal of Nervous and Mental Disease schrieb er gemeinsam mit seiner Fachkollegin Gertrude L. Cannon:

It has been shown that the laws governing the transmission of traits by heredity, as established by Mendel, hold good not only for plants and the lower animals, but also for man, at least as regards certain characters, such as color of hair and color of eyes. In view of this fact our problem has assumed for us a more definite form. It is simply: Are any of the forms of nervous and mental disease transmitted from generation to generation in accordance with the Mendelian laws? (Cannon/Rosanoff 1911: 272) 


\begin{tabular}{|c|c|c|c|c|c|c|c|c|c|c|c|}
\hline & & \multicolumn{2}{|c|}{ HuRst } & \multicolumn{2}{|c|}{$\begin{array}{c}\text { DAVEN- } \\
\text { PORT }\end{array}$} & \multicolumn{2}{|c|}{$\begin{array}{c}\text { HolMes \& } \\
\text { LOOMIS }\end{array}$} & \multicolumn{2}{|c|}{ Total } & \multicolumn{2}{|c|}{ P'ORTION } \\
\hline One Parent & Other Parent & Blue & Pig't & Blue & $P i g^{\prime} t$ & Blue & Pig't & Blue & Pig't & Blue & Pig't \\
\hline pure blue & pure blue & 101 & 0 & 77 & $0^{1}$ & 51 & 1 & 229 & 1 & 99.5 & 0.5 \\
\hline pigmented $(P p)$ & blue & 137 & 121 & 428 & 506 & 89 & 85 & 654 & 712 & 48.0 & 52.0 \\
\hline pigmented (PP) & blue & 0 & 66 & 0 & 70 & & & 0 & 136 & 0 & 100 \\
\hline pigmented (Pp) & pigmented (Pp) & 18 & 45 & $98^{2}$ & 169 & 5 & 34 & 121 & 248 & 33 & 67 \\
\hline pigmented (PP) & pigmented (Pp) & 0 & 195 & 0 & 99 & & & 0 & $294 \mid$ & 0 & 100 \\
\hline
\end{tabular}

Abb. 1: Darstellung zur Vererbung der Augenfarbe (Davenport 1912: 32).
Wie Rosanoff sah auch Rüdin die Mendel'schen Gesetze als ein gesichertes Wissen an, das eine plausible Interpretation der Vererbung von Geistesund Nervenkrankheiten ermöglichte. In seiner frühen programmatischen Schrift von 1911 gab er zwar zu, dass man bei psychischen Störungen noch keine mendelistische Vererbungsweise mit Sicherheit angeben konnte, er drückte aber seine Zuversicht aus, dass die in diesem Arbeitsfeld vorgenommenen Forschungsbemühungen zu diesem Ergebnis gelangen würden (vgl. Rüdin 1911: 519f.). In einem Aufsatz von 1923, der in der Zeitschrift für die gesamte Neurologie und Psychiatrie erschien, leitete er die Mendel'sche Vererbung geistiger Eigenschaften von der Vererbung somatischer Merkmale $\mathrm{ab}$, da diese auch auf ein organisches Substrat zurückzuführen sein müssten: „Da die geistigen Funktionen von einer körperlichen Unterlage, vom Zentralnervensystem und von anderen Organen des Körpers abhängen, so werden ihre Anlagen denselben Vererbungsgesetzen gehorchen wie alle übrigen Anlagen." (Rüdin 1923: 459)

Rosanoff, der sich der Unterstützung Davenports erfreute und dessen beide Aufsätze aus dem Jahr 1911 kurz nach ihrem Erscheinen in den Fachzeitschriften auch im Bulletin des ERO abgedruckt wurden (Cannon/Rosanoff 1911), fügte sich mit seinen Forschungen zudem in den Kontext der frühen nordamerikanischen Eugenik ein, welche die Verhinderung der Fortpflanzung Erbkranker durch eine gezielte Sterilisierungs- und Heiratspolitik anstrebte. Das von Davenport gegründete ERO war explizit mit dem Ziel eröffnet worden, die Erforschung der menschlichen Vererbung in den Dienst eugenischer Ziele zu stellen.

Bei Rüdin, der zu den Mitbegründern der 1905 ins Leben gerufenen deutschen Gesellschaft für Rassenhygiene zählte, war die Formulierung genetischer Forschungskonzepte von vornherein durch rassenhygienische Ideale motiviert. Als Leiter der GDA verfolgte er das Ziel, mit den in seiner Forschungsabteilung produzierten Ergebnissen wissenschaftliche Grundlagen für gesundheits- und sozialpolitische Maßnahmen zu schaffen. Dabei vertrat 
er - wie viele seiner Zeitgenossen - die Auffassung, dass das kollektive Wohl des Volkes, der Nation oder der Rasse dem Wohl des Individuums übergeordnet sei (Roelcke 2002b). Bei ihrer zeitgleichen Anwendung der Mendel'schen Theorie teilten Rosanoff und Rüdin ein gemeinsames Ziel: Beiden ging es um den Nachweis der Mendel'schen Vererbung psychischer Störungen und gleichzeitig um die Motivierung und Begründung eugenischer Maßnahmen. Trotz der übereinstimmenden Zielsetzung ihrer Forschungen waren die von Rosanoff und Rüdin produzierten Ergebnisse erheblich verschieden. Dies lag nicht nur an einer unterschiedlichen Methodik, sondern und vor allem an entgegengesetzten Krankheitskonzepten.

\section{Aaron J. Rosanoff und die Mendel'sche Deutung der Vererbung psychischer Störungen}

Im Beitrag von Rosanoff mit Cannon von 1911 wird die Anwendung der Mendel'schen Theorie dargestellt. Dabei plädieren sie für eine gründliche und exakte Erforschung der Genealogien von Patienten und übten wegen der Unzuverlässigkeit der Anstaltsstatistik scharfe Kritik an der Praxis, diese als Hauptquelle für die Erforschung des Vererbungsfaktors bei psychischen Störungen zu nutzen. Seit dem frühen 19. Jahrhundert war die Sammlung der Stammbäume von Patienten ein wichtiger Bestandteil der psychiatrischen Praxis. Darauf basierend wurden Statistiken erstellt, in denen unter anderem prozentuale Angaben über die Anzahl der Erkrankungen im Familienkreis der Patienten gemacht wurden. Rosanoff und Cannon kritisieren diese Statistiken, da sie auf unsicheren und unvollständigen Informationen beruhten, denn man hatte sich an die von den Patienten selbst gegebenen Auskünfte über ihre Verwandten halten müssen. Für Davenport und seine Arbeitsgruppe am ERO sicherte erst der Einsatz von Feldforschern den Erhalt vollständiger und zuverlässiger Informationen. Denn Voraussetzung für die Auswertung von Daten über die menschliche Vererbung war deren intensive Zusammenarbeit mit den Probanden sowie ihren Verwandten. Dadurch sollten sie vor möglichen Gedächtnis- und Diagnosefehlern geschützt werden. Rosanoff und seine Mitarbeiter beobachteten ihre Patienten auch direkt, um einen weitreichenden Einblick in die Erkrankungen und den Zustand ihrer Verwandten zu gewinnen. Rosanoff und Cannon waren die ersten Psychiater, die bei ihren Forschungen über die Vererbung von Geisteskrankheiten auch auf Feldforscher zurückgriffen und sich diesbezüglich auf die am ERO entwickelte Arbeitsmethodik stützten. Anlässlich einer am Ende des im Bulletin des ERO wiederabgedruckten Artikels von Cannon und Rosanoff publizierten Diskussion, die im Anschluss an Rosanoffs Vortrag in der New York Neurological Society stattfand, hatte Davenport die zentrale Bedeutung der neuartigen Praxis der Erhebung von Informationen bei den 
Kranken und ihren Familien nochmals explizit hervorgehoben (Cannon/Rosanoff 1911: 9).

Die Anwendung der Mendel'schen Theorie durch Rosanoff fußte nicht nur auf einer neuen Erhebungspraxis von Informationen über die Vererbung von psychischen Störungen. Sie war gleichzeitig von einer bereits feststehenden Vorstellung über die Vererbung getragen. Wie bereits angedeutet, setzten Rosanoff und seine Mitarbeiter bei ihren Untersuchungen das Postulat voraus, dass psychische Störungen hinsichtlich der Vererbung keine unabhängigen Einheiten bildeten. Denn schon bevor Rosanoff die Auswertung der in seinem Krankenhaus gesammelten Stammbäume präsentierte, stellte er fest, dass alle erblichen Formen von Nerven- und Geisteskrankheiten außer der Chorea Huntington nicht unabhängig voneinander weitergegeben würden und als solche von der Vererbung eines nicht weiter definierten „neuropathic make-up“ herrührten:

\footnotetext{
One of the first facts that appeared in the study of the pedigrees was that any form of insanity or even all the forms of hereditary insanity do not constitute an independent hereditary character, but that they are closely related to imbecility, epilepsy, hysteria, and various mental eccentricities that are not unusually included under the designation insanity. In other words, the distinction between these conditions as clinical entities cannot, in the light of their manner of origin, be regarded as deeply essential. We find as manifestation of the neuropathic make-up in closely related persons cases of feeble-mindedness, convulsions in childhood from trivial causes or chronic epilepsy, cases of grave hysteria, various eccentricities, cases of dementia praecox, manic-depressive insanity, paranoic conditions, involutional psychoses and the like. (Cannon/Rosanoff 1911: 275)
}

Beim Nachweis der Mendel'schen Vererbung des „neuropathic make-up“ ging Rosanoff in zwei Schritten vor: Erstens ergab die Analyse seines Stammbaummaterials, dass "neuropathische Kinder“ "normale Eltern" haben konnten (vgl. Beispiel aus Abb. 2). Für den umgekehrten Fall, die Geburt von „normalen Kinder“ von „neuropathischen Eltern“ fand er keinen Beleg. Aus diesen Ergebnissen postulierte er den rezessiven Charakter des „neuropathic make-up“ (ebd.). In einem zweiten Schritt ermittelte er die Proportionsverhältnisse zwischen „normalen“ und „neuropathischen“ Nachkommen ent-

Abb. 2: Ausschnitt aus einer Stammbaumanalyse zum Nachweis der Mendel'schen Vererbung des „neuropathic makeup" (Cannon/Rosanoff 1911: 277) sprechend der in den Stammbäumen auftretenden Kreuzungen und verglich sie mit dem Mendel'schen Erbgang. Die Ähnlichkeit der Ergebnisse nahm er als Bestätigung für die Annahme einer rezessiven Vererbung der sogenannten neuropathischen Konstitution. Abbildung 2 zeigt das Beispiel eines Stammbaums, bei dem „neuropathische“ Kinder von „normalen“ Eltern

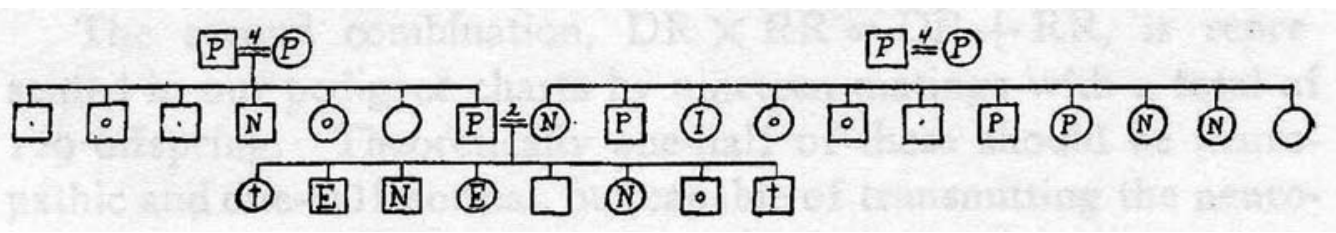


geboren wurden. Das Symbol „P“ bedeutet ein „normales“ Individuum mit einer „neuropathischen“ Nachkommenschaft, das „N“ steht für Schwachsinnigkeit, Hysterie oder weitere Formen einer "neuropathischen Konstitution“ und der Buchstabe E signalisiert das Auftreten von Epilepsie.

In Rosanoffs zweitem wichtigen Aufsatz von 1911, den er gemeinsam mit seiner Kollegin Florence I. Orr im American Journal of Insanity veröffentlichte, wertete er die Stammbäume sechzig weiterer Familien aus (Rosanoff/Orr 1911). Die Ergebnisse basierten auch hier auf einem Vergleich der ermittelten Proportionen zwischen "normalen“ und "neuropathischen“ Nachkommen mit den Prozentsätzen, die nach den Mendel'schen Erbgesetzen zu erwarten gewesen waren. Er wertete sie als Beleg für die rezessive Vererbung der „neuropathischen Konstitution“ (ebd.: 228). Das Vorkommen verschiedener "neuropathischer" Zustände in den von ihm gesammelten Stammbäumen interpretiert er nun als Ausdruck verschiedener Grade von Rezessivität. So seien einige Zustände weniger rezessiv als andere. Von der Beobachtung der Nachkommen eines Epileptikers und einer depressiven Frau leitete er zum Beispiel die Erkenntnis ab, dass heilbare Psychosen zwar rezessiv seien, sich zur Epilepsie jedoch dominant verhielten. Dieser Befund sollte aus seiner Sicht nicht zum Anlass genommen werden, die These der „neuropathischen Konstitution“ als ätiologische Einheit zu relativieren. Vielmehr betonte Rosanoff die Zusammengehörigkeit neuropathischer Zustände: Ihre klinischen Manifestationen seien zwar von der Persönlichkeit und der Umwelt geprägt, sie würden aber auf derselben Anlage basieren.

\section{Ernst Rüdin und die Mendel'sche Deutung der Vererbung psychischer Störungen}

Seit den 1910er Jahren arbeitete Rüdin an der Etablierung eines neuen psychiatrisch-humangenetischen Forschungskonzeptes. In seiner bereits erwähnten programmatischen Schrift von 1911 setzte er sich mit dem damaligen Stand der Vererbungsforschung auseinander und beschrieb eine neuartige Vorgehensweise zur Erforschung der genetisch-ätiologischen Mechanismen psychischer Störungen. Wie Rosanoff strebte Rüdin eine genauere und vollständigere Erforschung der Vererbung psychischer Störungen bei Blutsverwandten an. Im Gegensatz zu diesem wandte er sich allerdings gegen die Form der genealogischen Forschung, die etwa zwanzig Jahre zuvor eine starke Forschungsrichtung in der deutschen Psychiatrie einnahm. Sie war durch eine kasuistische Ausrichtung und ein primäres Interesse an den erblichen Beziehungen zwischen Nerven- und Geisteskrankheiten gekennzeichnet. Da dieser genealogische Forschungszweig sich auf isolierte Beispiele der familiären Häufung von Krankheiten konzentrierte, betrachtete Rüdin ihn als ungeeignet, um eine allgemeine Aussage über die Vererbungs- 
weise von Krankheiten zu treffen. Er sah die Aufklärung der Vererbung von Geisteskrankheiten wegen ihrer einseitigen Berücksichtigung schwer belasteter Familien als gescheitert an und plädierte für die statistische Analyse der generellen Verbreitung von einheitlich diagnostizierten Merkmalen.

Das von Rüdin entwickelte Forschungskonzept baute auf der Anwendung des von dem Mediziner und Statistiker Wilhelm Weinberg erarbeiteten Verfahrens zur Auswertung humangenetischer Daten auf. Weinberg hatte sich bereits seit 1900 mit den mathematischen Problemen genetischer Untersuchungen auseinandergesetzt und war zu der Erkenntnis gelangt, dass bei der Erhebung humangenetischer Daten eine einseitige Auslese der Erkrankten stattfindet. In das Blickfeld der Forscher waren immer wieder Familien mit einer größeren Anzahl von Kranken geraten. Darüber hinaus wurden bei der Untersuchung von einzelnen Familien die krankheitsfreien Geschwister nicht selten außer Acht gelassen. Um diese Probleme zu beheben, erarbeitete Weinberg 1912 ein mathematisches Verfahren, das er Probanden- und Geschwistermethode nannte. Damit sollte der irreführende Mangel an gesunden Individuen in den untersuchten Geschwisterserien korrigiert werden. ${ }^{4}$

In seiner ersten großen Forschungsarbeit zur Vererbung der Dementia praecox, die etwa zeitgleich mit der Formulierung seines theoretischen Forschungskonzeptes in Angriff genommen wurde (Weber 1993: 109), griff Rüdin auf Weinbergs Methode zurück. Erst 1916 präsentierte er in einer größeren Monographie seine Ergebnisse (Rüdin 1916). Nach Weinbergs Probandenmethode errechnete er die Häufigkeit von Dementia praecox und anderen Psychosen unter den Nachkommen gesunder sowie an Dementia praecox erkrankter Eltern. Er war dabei von 700 möglichst auslesefrei gesammelten und vollständig gezählten Krankheitsfällen ausgegangen, die in München seit Bestehen einer spezifischen Versorgung psychiatrischer Patienten diagnostiziert worden waren. Die Diagnosen folgten gemäß der Nosologie Kraepelins. Rüdins Untersuchung schloss alle Geschwister und Eltern der Patienten ein.

Im Gegensatz zu den Ergebnissen anderer Arbeiten, die höhere Zahlen angaben, kam Rüdin zu dem Ergebnis, dass unter den Geschwistern Erkrankter mit gesunden Eltern die Dementia praecox mit einer Häufigkeit von 4,48\% und andere Psychosen mit 4,12\% auftraten. Angesichts des Umstands, dass der Dementia praecox-Prozentsatz unter den Nachkommen gesunder Eltern nahe bei einem Sechzehntel lag, stellte er die Hypothese auf, dass die Erkrankung einem dihybriden Kreuzungs-Modus folge, wonach zwei konkurrierende Merkmalspaare in Aktion treten würden (Rüdin 1916: 163). Den eigentlichen Nachweis der rezessiven Vererbung konnte er nicht vorlegen. Rüdins Arbeit erfreute sich trotzdem großer Aufmerksamkeit und übte eine Vorbildfunktion aus. Seine Studie wurde im internationalen Kontext als Pionierleistung angesehen (Roelcke 2007). Im ersten englischen Handbuch zur Humangenetik, das im Jahr 1934 erschien, bezeichnete Aubrey Lewis, eine 
in der Mitte des 20. Jahrhunderts führende Persönlichkeit der britischen Psychiatrie, die Forschungsarbeit von Rüdin sogar als richtungsweisend für die Entwicklung der psychiatrischen Genetik:

Während der letzten zwanzig Jahre hat sorgfältige Arbeit zwei Vorwürfe gegenüber der psychiatrischen Genetik beseitigt: Daß dies schlechte Psychiatrie, und daß es schlechte Genetik sei. Dies ist einigen Männern gutzuschreiben, unter denen Rüdin der führende ist. Seine Untersuchungen waren der Ausgangspunkt und das Modell für fast alles Wertvolle, was in diesem Arbeitsfeld bisher geleistet wurde. (Lewis 1934: 87, zitiert nach Roelcke 2007: 178)

\section{Zur Divergenz der Mendel'schen Deutungspraxis}

Während Rosanoff an der seit der zweiten Hälfte des 19. Jahrhunderts verbreiteten Theorie der polymorphen Vererbung der Geisteskrankheiten festhielt und sich mit seiner Hinwendung zum Mendelismus um ihren Nachweis bemühte, war Rüdin bestrebt, mithilfe der Mendel'schen Theorie die These der spezifisch genetischen Ursache der von Kraepelin umschriebenen klinischen Krankheitseinheiten zu untermauern. Insofern war seine Anwendung der Mendel'schen Theorie von der polymorphen Theorie weiter entfernt. Die unterschiedliche Anwendung der Mendel'schen Theorie in der nordamerikanischen und deutschsprachigen Psychiatrie lässt sich damit weitgehend - wie herausgearbeitet - auf unterschiedliche Krankheitskonzepte zurückführen. Schon im ausgehenden 19. Jahrhundert hatte die deutschsprachige Psychiatrie damit begonnen, sich von der Theorie des Polymorphismus zu distanzieren. Seit Mitte der 1880er Jahre wurde in der Fachwelt über die Gültigkeit der Lehre von August Morel zur Transformation und Degeneration von Geisteskrankheiten debattiert. Nicht nur die Annahme der fortschreitenden Entartung des Menschengeschlechts bildete einen strittigen Punkt, sondern auch und vor allem die erfolgreiche These des Polymorphismus wurde zunehmend hinterfragt. Mehrere jüngere Psychiater wie Emil Sioli (Bunzlau), Wilhelm Strohmeyer (Jena) oder R. Foerster (Bonn) gelangten durch ihre klinischen Studien zunehmend zu der Einsicht, dass die Geisteskrankheiten der Vorfahren die Tendenz hatten, identisch oder ähnlich bei den Nachkommen aufzutreten. ${ }^{5}$ Vor allem waren sie der Meinung, dass bestimmte Krankheiten wie Dementia praecox und manisch-depressives Irresein sich offenbar ausschlossen. Mit dem Durchbruch der Kraepelin'schen Krankheitslehre wurde die zunehmend verbreitete Ansicht, dass Nervenund Geisteskrankheiten sich identisch vererben, erheblich gestärkt, auch wenn die Idee des Polymorphismus von Nerven- und Geisteskrankheiten nicht ganz aufgegeben wurde.

Um seine Grundthese der gemeinsamen Ätiologie psychischer Störungen zu untermauern, stützte sich Rosanoff auf eine retrospektive Darstellung des nosologischen Fortschritts. Bemerkenswerterweise machte er in diesem $\mathrm{Zu}$ - 
sammenhang auf die zentrale Bedeutung Kraepelins aufmerksam. Dessen Erfolg auf dem Gebiet der Nosologie beruhe auf der Zusammenführung verschiedener Symptome in ein und dieselbe Kategorie. Kraepelin sei nämlich derjenige, der zur Vereinfachung der Nosologie beigetragen habe, indem er die Zusammengehörigkeit sehr verschiedener Symptome erkannte:

More recently Kraepelin has shown that certain depressions, manias, circular and mixed states are but various phases of the same underlying constitutional disorder. [...] Similarly, in one immense group, under the general heading of dementia praecox we now, following Kraepelin, include such widely contrasted conditions as simple hebephrenia, catatonia, and délire chronique à évolution systématique - conditions which were long regarded as independent clinical entities. (Rosanoff/Orr 1911: 233)

Vor diesem Hintergrund schilderte Rosanoff seine Auffassung über die $\mathrm{Zu}$ sammengehörigkeit neuropathischer Zustände sogar als eine Erweiterung von Kraepelins Ideen: „Some of the data furnished by our material seem to indicate the necessity for a still further extension of this conception." (Ebd.)

Während Rosanoff sich auf Kraepelin stützte, um seine These der polymorphen Vererbung der Geistes- und Nervenkrankheiten zu untermauern, wies Rüdin auf Kraepelin hin, um die spezifische Erbbiologie psychischer Störungen zu begründen. Genauso wie Rosanoff seine These zu bekräftigen versuchte, war Rüdin bemüht, vermehrt Argumente dagegen zu unterbreiten. Sein Hauptargument basierte auf dem statistischen Häufigkeitsnachweis, dass unterschiedliche Geisteskrankheiten nicht häufiger als üblich in denselben Familien vorkamen:

\footnotetext{
Es gibt manche Psychiater, die an einen inneren, erbgenetischen Zusammenhang z.B. der Dementia praecox und des manisch-depressiven Irreseins glauben. Man findet diese Psychosen auch mitunter in der gleichen Familie. Aber beide Krankheiten sind an und für sich ziemlich häufig. Ihr nicht allzu seltenes Zusammenvorkommen ist daher nicht erstaunlich. Daß es sich aber nur um zufälliges Zusammentreffen handelt, kann nur eine statistische Untersuchung feststellen, d.h. z.B. ein Vergleich der Zahl der Dementia praecox-Geschwister von andersartigen Probanden. Nach meinen bisherigen Auszählungen der an Dementia praecox leidenden Geschwister der verschiedensten psychotischen Probandenarten, z.B. auch der als exogen entstanden betrachteten Paralyse, ergaben sich keine großen Abweichungen der Dementia praecox-Ziffern. (Rüdin 1923: 478)
}

Darüber hinaus wies Rüdin auf die Bedeutung der Inzucht und die Wirkungen einer ungünstigen Heiratsauslese bei den Nerven- und Geisteskranken hin. Seiner Ansicht nach könnten auch Diagnosefehler die Annahme des vermeintlichen Auftretens unterschiedlicher Krankheiten bei denselben Blutsverwandten bedingen. Aus diesem Grund legte Rüdin besonderen Wert auf die Gewinnung eines diagnostisch gesicherten und einheitlichen Untersuchungsmaterials. Im Rahmen seiner Forschungsarbeit zur Vererbung der Dementia praecox bezog sich Rüdin auf eindeutige Krankheitsfälle, die den von Kraepelin aufgestellten diagnostischen Kriterien entsprachen (ebd.: 24). Rüdin ging es im Kern dabei um die Aufdeckung des Vererbungsvorgangs des von Emil Kraepelin beschriebenen Leidens. 


\section{Zwischen Ablehnung und Weiterführung Mendel'scher Forschungsansätze in der Psychiatrie}

Nicht nur in Deutschland, sondern auch in Großbritannien und den USA stieß die Einführung des Mendelismus in die Psychiatrie in der Version von Rosanoff und Davenport zunächst auf große Ablehnung. David Heron, ein Schüler des britischen Biometrikers Karl Pearson und Forschungsassistent am Londoner Galton Laboratory, bezog bereits 1913 Stellung gegen die nordamerikanischen Arbeits- und Deutungsmethoden. Herons Kritik (1913: 3) gründete sich zunächst auf die Befürchtung, dass fehlgeleitete Forschungsansätze für die Weiterentwicklung eugenischer Forschung als angewandte Wissenschaft extrem schädlich seien. In Abgrenzung zu Davenports und David F. Weeks' Arbeiten über Epilepsie und Schwachsinn machte er darauf aufmerksam, dass die von ihnen dargestellten Proportionen zwischen "normalen“ und „belasteten“ Kindern weder mit den von ihnen in Form von Tabellen aufgestellten Daten noch mit den Mendel'schen Proportionsverhältnissen übereinstimmten. Heron nahm diese Studie zum Anlass einer Generalkritik gegen Davenports Forschung. Sie sei durch eine nachlässige Präsentation der Daten, unpräzise Analysemethoden, überstürzte Schlussfolgerungen und wechselnde Ansichten gekennzeichnet (ebd.: 5). An Rosanoffs und Orrs Arbeiten kritisierte er das diffuse Konzept der „neuropathischen Konstitution“, die sehr verschiedene Zustände umfasse:

\footnotetext{
The use of the term ,insanity 'in the titles of these two papers is very misleading; only a comparatively small proportion of the affected individuals are actually insane. These papers deal, not with the inheritance of insanity, but with the inheritance of what the authors call a "neuropathic" condition which is so comprehensive that it is a matter of surprise that there are any "normal" individuals at all. It is indeed, a fortunate circumstance that the Mendelian theory requires the presence of some normal individuals. (Ebd.: 38)
}

Ihre Vorgehensweise sei von einer Logik beherrscht, die die Forschungsergebnisse um jeden Preis und entgegen der eigentlichen empirischen Befunde mit der Theorie übereinstimmen lasse. Die Schlussfolgerungen von Rosanoff und Orr seien anhand der von ihm ermittelten Daten nicht nachvollziehbar. Heron machte zum Beispiel darauf aufmerksam, dass danach nicht alle der von zwei neuropathischen Eltern zur Welt gebrachten Kinder ebenfalls neuropathisch seien - was als Befund im klaren Gegensatz zu den Voraussetzungen stand (ebd.: 40).

Der Bostoner Psychiater Abraham Myerson, der sich mit Stammbaumforschung befasste, argumentierte 1917 in seiner Kritik an Davenports und Rosanoffs Forschungen in gleicher Weise. Die Schlussfolgerung einer zwingend bestehenden Übereinstimmung der Befunde mit der Theorie war für ihn unannehmbar: „[Davenport and Rosanoff, A.C.] have worked entirely from the standpoint of Mendelism, and their efforts seem to me to be directed not so much to discover the laws of the transmission of insanity as to 
fit the facts to Mendelian theory." (Myerson 1917: 359) Außerdem war Myerson der Überzeugung, dass die Anwendung der Mendel'schen Theorie im Bereich der Psychiatrie verfrüht sei, weil bisher kaum bei einem einfachen menschlichen Merkmal eine Mendel'sche Vererbung nachgewiesen worden sei. Vor diesem Hintergrund hielt es Myerson für besonders anmaßend, eine Mendel'sche Vererbung von komplexen Merkmalen wie psychischen Störungen anzunehmen. Vor allem aus diesem Grund richtete er sich gegen Rosanoffs Konzept der neuropathischen Konstitution als einer erbbiologischen Einheit: „To assume then that the vast range of the psychoses is related to a unit determiner or a group of determiners acting as a unit is, to say the least, premature." (Ebd.: 360) Angesichts des Entwicklungsstandes der experimentellen Vererbungsforschung empfahl deshalb Myerson, von der statistischen Auswertung unvollständiger Daten abzusehen und sich stattdessen der exakten Erforschung möglichst vollständiger Stammbäume zu widmen. Nur dadurch sei die Entdeckung von Tendenzen der pathologischen Vererbung möglich (Myerson 1925: 282). Er plädierte damit für eine Rückkehr zur Stammbaumforschung, die in Deutschland ein Jahrzehnt vor der Jahrhundertwende eine besondere Entfaltung erlebt hatte. In einer Serie von zwei Aufsätzen, die 1917 und 1918 im American Journal of Insanity mit dem Titel „Psychiatric Family Studies“ erschienen, breitete Myerson die Ergebnisse seiner eigenen Stammbaumuntersuchungen an den Krankenakten des Taunton State Hospital (Massachusetts) und seiner Feldforschung über die internierten Patienten und ihre Verwandten aus. Darauf basierend rekonstruierte er die einzelnen Stammbäume der versorgten Geisteskranken, die er in seiner Serie mit ausführlichen Kommentaren reproduzierte. In Abbildung 3 ist am Beispiel eines der vielen von Myerson in seinem Aufsatz von 1917 wiederabgedruckten Stammbäume die Vererbung des manischdepressiven Irreseins bei der Nachkommenschaft eines an dieser Störung erkrankten Mannes zu sehen. Der Buchstabe „I“ weist auf das Vorkommen einer Geisteskrankheit. „T.B.“ bedeutet Tuberculosis und „N“ bedeutet normal. In seinem Kommentar zum Stammbaum stellt Myerson fest, dass die Symptome des kranken Sohnes des beobachteten Manisch-Depressiven nicht gleich ausfallen, aber dass es sich hierbei eindeutig um einen Fall von manisch-depressivem Irresein handelt.

Im Gegensatz zu Myerson hatte Rüdin die weitere Erforschung von einzelnen Stammbäumen abgelehnt, da nach seiner Auffassung auf dieser Grundlage kein Nachweis von Vererbungsgesetzen möglich sei. Er plädierte dagegen für die sachkundige Anwendung statistischer Methoden an einem möglichst auslesefrei gesammelten Untersuchungsmaterial. Obwohl seine Anwendung der Mendel'schen Theorie durch die Aufdeckung einer Gesetzmäßigkeit der Vererbung von psychischen Störungen motiviert war, musste sich Rüdin sehr schnell (wie seine Forschungsarbeiten zur Vererbung der Dementia praecox zeigen) eingestehen, dass dieses Ziel vorerst nicht erreicht 


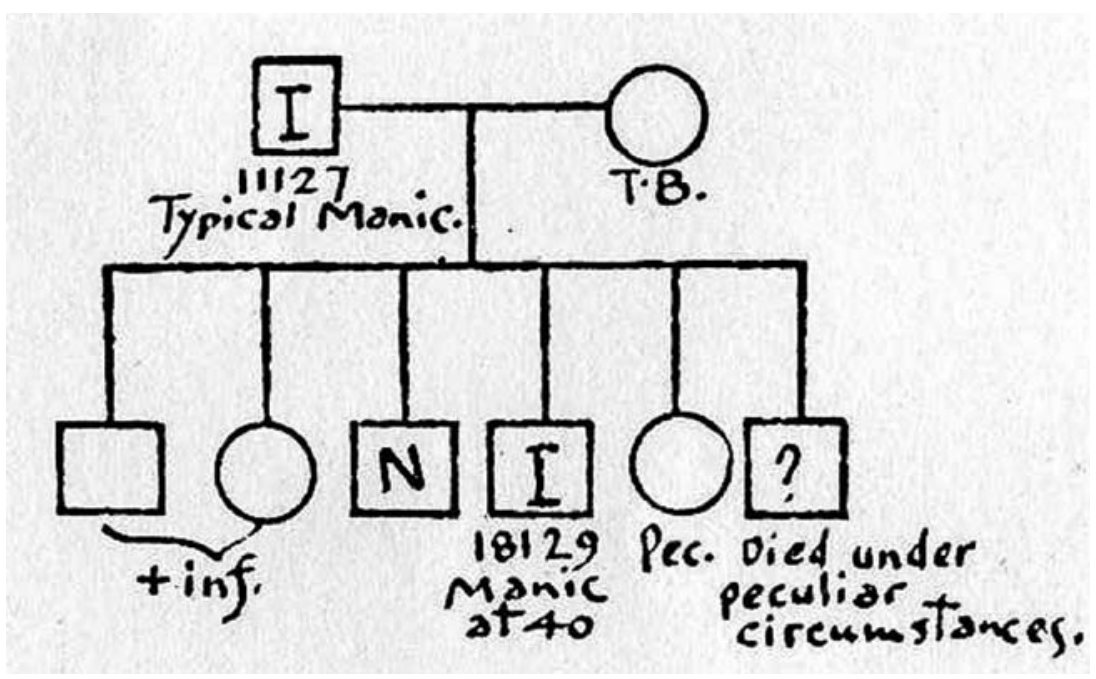

Abb. 3: Die Vererbung des manisch-depressiven Irreseins in der Familiengruppe Nr. 44 (Myerson 1918: 423)

werden konnte. Infolgedessen entwarf er ein Programm zur empirischen Ermittlung von Erbprognosen. Statt nun weiter nach einer mendelistischen Gesetzmäßigkeit zu suchen, verlangte Rüdin jetzt, die Erkrankungswahrscheinlichkeit einer Person je nach ihrem Verwandtschaftsgrad zu einem erblich erkrankten Familienangehörigen zu berechnen. Er wollte noch nicht feststellbare Kausalbeziehungen durch statistische Wahrscheinlichkeiten über die familiären Häufungen psychischer Störungen ersetzen. Die Weichenstellungen für das Programm der sogenannten empirischen Erbprognose erfolgten bereits im Rahmen seiner Dementia praecox-Studie. Rüdin war der Nachweis der rezessiven Vererbung der Erkrankung zwar nicht gelungen aber sein Ergebnis, wonach die Dementia praecox-Inzidenz unter den Nachkommen Dementia praecox-freier Eltern nahe bei einem Sechzehntel lag, bildete dennoch im Hinblick auf die rassenhygienische Praxis ein sehr nützliches Wissen. Denn mit der empirischen Erbprognose war das Ziel einer eugenisch motivierten Verhinderung der Verbreitung von Geisteskrankheiten unmittelbar verknüpft. Nachdem die ersten Überlegungen zur Aufstellung empirischer Erbprognosen im Kontext der Dementia praecox-Studie entstanden waren, wurde Rüdins Forschungsansatz im Laufe der 1920er Jahre mit großem Einsatz in die Praxis umgesetzt. An der GDA wurde eine Vielzahl von Forschungsprojekten in Angriff genommen, die das Ziel verfolgten, derartige Prognosen zu ermitteln. ${ }^{6}$ Das Programm der empirischen Erbprognose, das zu einer Verdrängung der Weinberg'schen Mathematik und ihrer sogar vornehmlich von Rüdin selber zugewiesenen Bedeutung führte, stellte schließlich seit Ende der 1920er Jahre nicht zuletzt im Hinblick auf sein eugenisches Anwendungspotential den mächtigsten Ansatz zur Untersuchung humangenetischer Daten dar (Mazumdar 1996: 644-657). 
Im Zeichen des zunehmenden politischen Einflusses eugenischer Kosten-Nutzen-Rechnungen war Rüdin seit Ende der 1920er Jahre immer mehr darum bemüht, die zentrale Bedeutung seines neuen Forschungsansatzes für die rassenhygienische Praxis zu betonen (Rüdin 1928, 1929, 1930, 1933). Texte zur Erläuterung der neuen Forschungsrichtung, aber auch erste Ergebnisse wurden veröffentlicht. ${ }^{7}$ Nach der Machtübernahme durch die Nationalsozialisten wurde das Programm der empirischen Erbprognose durch eine kräftige Förderung weiter ausgebaut. ${ }^{8}$ Sowohl feste Mitarbeiter als auch Gastwissenschaftler und Doktoranden setzten die Berechnungen über genauere Häufigkeitsziffern für verschiedene psychische Störungen, Verwandtschaftsgrade und geographische Regionen an der GDA fort. ${ }^{9}$ Dabei wurden der NS-Politik der Erb- und Rassenpflege brauchbare Daten zur Verfügung gestellt. Noch 1938 drängte Rüdin auf die unmittelbare Anwendung der durch das Programm der „empirischen Erbprognose“ aufgezeigten Ergebnisse:

Leitender Gesichtspunkt bei allen Zwillings- und empirisch erbprognostischen Untersuchungen, die innerhalb und außerhalb des Münchner Institutes vorgenommen werden mussten, war und ist die Anschauung, dass wir nicht warten können, bis die Gelehrten der ganzen Erde 100prozentig für ihre Volkserbkrankheiten sich auf die Annahme eines bestimmten Mendelschen Erbmodus geeinigt haben. Denn über diesem Warten auf unbestimmte Zeit würden die Kulturvölker unterdessen zugrunde gehen. (Rüdin 1938: 18)

Die an der GDA ermittelten Erbprognosen für verschiedene Störungen bildeten ein wichtiges Hilfsmittel bei der Umsetzung der NS-Rassenhygiene. Aufgrund seiner engen Kooperation mit den NS-Machtzentren und Gesundheitsbehörden erhoffte sich Rüdin die Umsetzung der Forschungsergebnisse seiner Arbeitsgruppe in Form rassenhygienischer Maßnahmen. Im 1934 erschienenen offiziellen Kommentar zum Gesetz zur Verhütung erbkranken Nachwuchses, den Rüdin mit dem Ministerialdirektor im Innenministerium Arthur Gütt und dem Juristen Falk Ruttke verfasst hatte, wird wiederholt auf "systematische erbprognostische Untersuchungen“ zur konkreten Feststellung der Erblichkeit in Einzelfällen hingewiesen. Die von Rüdin formulierte und an der GDA praktizierte Methode der empirischen Erbprognose war bereits zu Beginn der NS-Diktatur zum offiziellen Bestandteil der staatlichen Bevölkerungspolitik geworden. ${ }^{10}$

\section{Anmerkungen}

1 Damit stand er an der Spitze einer der ersten Arbeitsgruppen zur psychiatrischen Genetik, die bereits im Laufe der 1920er Jahre eine internationale Vormachtstellung genoss (Roelcke 2007: 177, Weber 1993: 114-124).

2 Sowohl Davenport als auch Rüdin waren in den Führungsgremien der International Federation of Eugenic Organizations (IFEO) vertreten, die 1925 aus einer Permanent International Eugenics Commission hervorging und das Ziel einer international organisierten Ras- 
senforschung verfolgte. 1932 wurde Rüdin zum Nachfolger von Davenport als Präsident der IFEO gewählt. Davenport und Rüdin, die in Briefkontakt standen, waren beide sehr um eine Internationalisierung der eugenischen Bewegung bemüht. Siehe Kühl 1997: 137; Rüdin an Davenport, 8. September 1932 und Davenport an Rüdin, 19. September 1932, Max-PlanckInstitut für Psychiatrie, Historisches Archiv, München, GDA 34.

3 Alkaptonurie ist eine Funktionsstörung des Aminosäurestoffwechsels und bei der Brachydaktilie sind die Phalangen des Menschen verkürzt, vgl. Garrod 1902, Farabee 1905, Becker 1968: 89, Davenport/Davenport 1907, 1910.

4 Zur Entstehung und Erläuterung der Probanden- und Geschwistermethode siehe Weinberg 1907, 1910, 1911, 1912 und 1913.

5 Siehe z. B. Sioli 1885, Krauss 1903, Strohmayer 1904, Foerster 1907, Schlub 1909, Damköhler 1910.

6 Siehe z. B. Juda 1928, Luxenburger 1928, Schulz 1926, Banse 1929.

7 Siehe Juda 1928, Walker 1929, Konstantinu 1930, Luxenburger 1931, 1932.

8 Zur finanziellen Förderung von Rüdins Arbeitsgruppe vor und nach 1933 vgl. Roelcke 2006.

9 Siehe z. B. Juda 1935, Riedel 1937, Slater 1938, Schulz 1938, Conrad 1938.

10 siehe dazu Roelcke 2002b.

\section{Literatur}

Ackerknecht, Erwin, 1957. Kurze Geschichte der Psychiatrie. Stuttgart: Ferdinand Enke Verlag. Allen, Garland E., 1986. The Eugenics Record Office at Cold Spring Harbor, 1910-1940. An Essay in Institutional History. Osiris, 2, 225-264.

Banse, Johannes, 1929. Zum Problem der Erbprognosebestimmung. Die Erkrankungsaussichten der Vettern und Basen von Manisch-Depressiven. Zeitschrift für die gesamte Neurologie und Psychiatrie, 119, 576-612.

Bateson, William, 1906. An Address on Mendelian Heredity and its Application to Man. Brain, 29, $157-179$.

Cannon, Gertrude L./Rosanoff Aaron J., 1911. Preliminary Report of a Study of Heredity in Insanity in the Light of the Mendelian Laws. The Journal of Nervous and Mental Disease, 38, 272-280 [wiederabgedruckt mit einem Diskussionsbericht in Bulletin, Eugenics Record Office, 3, 1-11].

Conrad, Klaus, 1938. Übersicht über auslesefreie Untersuchungen in der Verwandtschaft von Epileptikern. Zeitschrift für psychiatrische Hygiene, 10, 167-184.

Damköhler, 1910. Vererbung von Geisteskrankheiten. Allgemeine Zeitschrift für Psychiatrie, 67, 643-646.

Davenport, Charles Benedict/Davenport, G., 1907. Heredity of Eye-Color in Man. Science, 26, 589-592.

Davenport, Charles Benedict/Davenport, G., 1910. Heredity of Skin Pigment in Man. The American Naturalist, 44, 705-731.

Davenport, Charles Benedict/Weeks, David F., 1911. A First Study of Inheritance of Epilepsy. Bulletin des Eugenics Record Office, 4, 1-80.

Davenport, Charles Benedict, 1912. Heredity in Relation to Eugenics. London: Williams and Norgate.

Davenport, Charles/Muncey, Elizabeth B., 1916. Huntington's Chorea in Relation to Heredity and Eugenics. American Journal of Insanity, 73, 204-207.

Engstrom, Eric J., 1990. Emil Kraepelin. Leben und Werk des Psychiaters im Spannungsfeld zwischen positivistischer Wissenschaft und Irrationalität. Internetquelle: http://www.engstrom. de/Kraepelinbiography.pdf [zugegriffen am 6. Mai 2004].

Farabee, William Curtis, 1905. Inheritance of Digital Malformations in Man. Papers of the Peabody Museum of American Archaeology and Ethnology. Harvard University, 3, Nr. 3, Cambridge. 
Foerster, R., 1907. Über die klinischen Formen der Psychosen bei direkter Erblichkeit. Allgemeine Zeitschrift für Psychiatrie, 64, 176-179.

Garrod, Archibald E., 1902. The Incidence of Alkaptonuria. A Study in Chemical Individuality. Lancet, 2, 1616-1620.

Gausemeier, Bernd, 2005. From Pedigree to Database. Genealogy and Human Heredity in Germany, 1890-1914. In: A Cultural History of Heredity III: 19th and early 20th Centuries. Preprint 294. Berlin: Max-Planck-Institut für Wissenschaftsgeschichte.

Heron, David, 1913. Mendelism and the Problem of Mental Defect. I. A Criticism of Recent American Work. Cambridge: Cambridge University Press.

Juda, Adele, 1928. Zum Problem der empirischen Erbprognosebestimmung. Über die Erkrankungsaussichten der Enkel Schizophrener. Zeitschrift für die gesamte Neurologie und Psychiatrie, 113, 477-517.

Juda, Adele, 1935. Psychiatrisch-genealogische Untersuchungen an 147 Hilfsschülern. Beitrag zur Frage: Sind alle Hilfsschüler schwachsinnig? Zeitschrift für psychische Hygiene, 8, 103125.

Konstantinu, Thrasiwulos, 1930. Zum Problem der Erbprognosebestimmung. Die Erkrankungsaussichten der Neffen und Nichten, Großneffen und Großnichten von Schizophrenen Thüringens. Zeitschrift für die gesamte Neurologie und Psychiatrie, 125, 103-133.

Krauss, Theodor, 1903. Über die Vererbung von Geisteskrankheiten. Allgemeine Zeitschrift für Psychiatrie, 60, 224-231.

Kühl, Stefan, 1997. Die Internationale der Rassisten. Aufstieg und Niedergang der internationalen Bewegung für Eugenik und Rassenhygiene im 20. Jahrhundert. Frankfurt: Campus.

Lewis, Aubrey, 1934. Inheritance of Mental Disorders. In: Charles P. Blacker, Hg., The Chances of Morbid Inheritance. London: H. K. Lewis, 86-133.

Luxenburger, Hans, 1928. Demographische und psychiatrische Untersuchungen in den engeren biologischen Familien von Paralytikerehegatten. Versuch einer Belastungsstatistik der Durchschnittsbevölkerung. Zeitschrift für die gesamte Neurologie und Psychiatrie, 112, 331-491.

Luxenburger, Hans, 1931. Zur Erbprognose bei rezessivem Erbgange. Eugenik - Erblehre - Erbpflege, 2, 60-62.

Luxenburger, Hans, 1932. Erbprognose und praktische Eugenik im cyclothymen Kreise. Der Nervenarzt, 5, 505-518.

Mazumdar, Pauline M. H., 1996. Two Models for Human Genetics. Blood Grouping and Psychiatry in Germany between the World Wars. Bulletin of the History of Medicine, 70, 609657.

Myerson, Abraham, 1917. Psychiatric Family Studies. American Journal of Insanity, 74, 355486.

Myerson, Abraham, 1918. Psychiatric Family Studies, paper no 2. American Journal of Insanity, 74, 497-554.

Myerson, Abraham, 1925. The Inheritance of Mental diseases. Baltimore: Williams and Wilkins Company.

Olby, Robert, 1994. The Path to the Double-Helix. New York: Dover Publications.

Rheinberger, Hans-Jörg, 2006. Epistemologie des Konkreten. Studien zur Geschichte der modernen Biologie. Frankfurt a. M.: Suhrkamp.

Riedel, Heinz, 1937. Zur empirischen Erbprognose der Psychopathie (Untersuchungen an Kindern von Psychopathen). Zeitschrift für die gesamte Neurologie und Psychiatrie, 159, 597-667.

Roelcke, Volker, 2002a. Die Entwicklung der Psychiatrie zwischen 1880 und 1932. Theoriebildung, Institutionen, Interaktionen mit zeitgenössischer Wissenschafts- und Sozialpolitik. In: Rudolf vom Bruch und Brigitte Kaderas, Hg., 2002, Wissenschaften und Wissenschaftspolitik. Bestandsaufnahmen zu Formationen, Brüchen und Kontinuitäten im Deutschland des 20. Jahrhunderts. Stuttgart: Steiner Verlag, 109-124.

Roelcke, Volker, 2002b. Programm und Praxis der psychiatrischen Genetik an der Deutschen Forschungsanstalt für Psychiatrie unter Ernst Rüdin. Zum Verhältnis von Wissenschaft, Politik und Rasse-Begriff vor und nach 1933. Medizinhistorisches Journal, 37, 21-55. 
Roelcke, Volker, 2003. Unterwegs zur Psychiatrie als Wissenschaft. Das Projekt einer „Irrenstatistik“ und Emil Kraepelins Neuformulierung der psychiatrischen Klassifikation. In: Eric J. Engstrom und Volker Roelcke, Hg., Psychiatrie im 19. Jahrhundert. Forschungen zur Geschichte von psychiatrischen Institutionen, Debatten und Praktiken im deutschen Sprachraum. Basel: Schwabe Verlag, 169-188.

Roelcke, Volker, 2006. Funding the Scientific Foundations of Race Policies. Ernst Rüdin and the Impact of Career Resources on Psychiatric Genetics, ca. 1910-1945. In: Wolfgang Eckart, Hg., Man, Medicine and the State. The Human Body as an Object of Government Sponsored Medical Research in the 20th Century. Stuttgart: Franz Steiner Verlag, 73-88.

Roelcke, Volker, 2007. Die Etablierung der psychiatrischen Genetik in Deutschland, Großbritannien und den USA, ca. 1910-1960. Zur untrennbaren Geschichte von Eugenik und Humangenetik. Acta Historica Leopoldina, 48, 173-190.

Rosanoff, Aaron J./Orr, Florence I., 1911. A Study in Insanity in the Light of the Mendelian Theory. American Journal of Insanity, 68, 221-261 [wiederabgedruckt in Bulletin, Eugenics Record Office, 5, 221-261].

Rüdin, Ernst, 1911. Einige Wege und Ziele der Familienforschung, mit Rücksicht auf die Psychiatrie. Zeitschrift für die gesamte Neurologie und Psychiatrie, 7, 487-585.

Rüdin, Ernst, 1916. Zur Vererbung und Neuentstehung der Dementia praecox. Berlin: Verlag von Julius Springer.

Rüdin, Ernst, 1923. Über Vererbung geistiger Störungen. Zeitschrift für die gesamte Neurologie und Psychiatrie, 81, 459-496.

Rüdin, Ernst, 1928. Über die Vorhersage von Geistesstörung in der Nachkommenschaft. Archiv für Rassen- und Gesellschaftsbiologie, 20, 394-407.

Rüdin, Ernst, 1929. Über psychiatrische Erbprognosebestimmung. Deutsche Medizinische Wochenschrift, 55, 1031-1033.

Rüdin, Ernst, 1930. Praktische Ergebnisse der psychiatrischen Erblichkeitsforschung. Archiv für Rassen- und Gesellschaftsbiologie, 24, 228-237.

Rüdin, Ernst 1933. Empirische Erbprognose. Archiv für Rassen- und Gesellschaftsbiologie, 27, 271-283.

Rüdin, Ernst, 1938. Die empirische Erbprognose, die Zwillingsmethode und die Sippenforschung in ihrer Bedeutung für die psychiatrische Erbforschung und für die Psychiatrie. Allgemeine Zeitschrift für Psychiatrie, 107, 3-20.

Rushton, Alan R., 1994. Genetics and Medicine in the United States 1800 to 1922. Baltimore/ London: The Johns Hopkins University Press.

Schlub, H. O. , 1909. Über Geisteskrankheit bei Geschwistern. Allgemeine Zeitschrift für Psychiatrie, 66, 514-541.

Schulz, Bruno, 1926. Zum Problem der Erbprognose-Bestimmung. Die Erkrankungsaussichten der Neffen und Nichten Schizophrener. Zeitschrift für die gesamte Neurologie und Psychiatrie, 102, 1-37.

Schulz, Bruno, 1938. Über die Möglichkeit des Auftretens von Kinderreihen mit Schizophrenen unter der Nachkommenschaft Schizophrener. Zeitschrift für die gesamte Neurologie und Psychiatrie, 162, 327-342.

Sioli, Emil, 1885. Über direkte Vererbung von Geisteskrankheiten. Archiv für Psychiatrie und Nervenkrankheiten, 16, 113-150.

Slater, Eliot, 1938. Zur Erbpathologie des manisch-depressiven Irreseins. Die Eltern und Kinder von Manisch-Depressiven. Zeitschrift für die gesamte Neurologie und Psychiatrie, 163, $1-47$.

State Eugenic Control, 1913. Bulletin 9. Cold Spring Harbor: Eugenics Record Office, 37-40 [Compiled by Charles B. Davenport].

Strohmayer, Wilhelm, 1904. Ziele und Wege der Erblichkeitsforschung in der Neuro- und Psychopathologie. Allgemeine Zeitschrift für Psychiatrie, 61, 355-369.

The Family-History Book, 1912. Bulletin 7. Cold Spring Harbor: Eugenics Record Office [ Compiled by Charles B. Davenport].

Walker, Heinrich, 1929. Zum Problem der empirischen Erbprognosebestimmung. Die Erkran- 
kungsaussichten der Neffen und Nichten sowie der Großneffen und Großnichten Basler Schizophrener. Zeitschrift für die gesamte Neurologie und Psychiatrie, 120, 100-120.

Weber, Matthias M., 1993. Ernst Rüdin. Eine kritische Biographie. Berlin: Springer Verlag.

Weinberg, Wilhelm, 1907. Verwandtenehe und Geisteskrankheit. Archiv für Rassen- und Gesellschaftsbiologie, 4, 471-475.

Weinberg, Wilhelm, 1910. Statistik und Vererbung in der Psychiatrie. Klinik für psychische und nervöse Krankheiten, 5, 34-43.

Weinberg, Wilhelm, 1911. Vererbungsforschung und Genealogie. Eine nachträgliche Kritik des Lorenzschen Lehrbuches. Archiv für Rassen- und Gesellschaftsbiologie, 8, 753-760.

Weinberg, Wilhelm, 1912. Weitere Beiträge zur Theorie der Vererbung. Archiv für Rassen- und Gesellschaftsbiologie, 9, 165-174, 694f.

Weinberg, Wilhelm, 1913. Auslesewirkungen bei biologisch-statistischen Problemen. Archiv für Rassen- und Gesellschaftsbiologie, 10, 417-451, 557-581.

\author{
Anne Cottebrune \\ Institut für Geschichte der Medizin der Universität Gießen \\ Jheringstr. 6 \\ D-35392 Gießen \\ E-Mail: anne.cottebrune@histor.med.uni-giessen.de
}

